\title{
Continuation of minimally invasive surgery in the COVID-19 pandemic
}

\author{
J. R. Huddy ${ }^{1}$ S. Rasheed ${ }^{2,3} \cdot$ H. S. Tilney ${ }^{1,3}$ \\ Received: 30 July 2020 / Accepted: 17 August 2020 / Published online: 26 August 2020 \\ (c) Springer Nature Switzerland AG 2020
}

Dear Sir,

We note with interest the evolving advice concerning the surgical management of colorectal conditions in the COVID19 pandemic from national bodies. We are concerned about the effects of variations in these guidelines over time, and their effect on patient outcomes.

Initial anxieties regarding the use of laparoscopy for colorectal cancer resections, as well as the suggestions that anastomoses should be avoided due to the risks of anastomotic leaks were based on the assumptions that the likelihood of COVID-related complications was high, but that the procedural urgency meant that the surgery should not be delayed. In hindsight, we feel that both of these assumptions, made with the best of intentions to minimise patient harm, are likely to have been exaggerated.

The use of laparoscopy in COVID-19 offers clear potential advantages to patients, including lower morbidity and shorter lengths of stay so that their interactions with a stressed healthcare service are minimised, reducing the risks of nosocomial viral transmission. In fact, it is the surgical team who bear the theoretical risks of COVID in this circumstance, but we believe that the use of appropriate personal protective equipment (PPE) and liberal testing of staff and patients will almost eliminate this risk [1]. To omit anastomosis and perform open surgery $[1,2]$ with a longer length of stay, seems unacceptable as the significant adverse consequences for our patients are not justified by small potential increased risks to staff, which can be offset almost entirely by appropriate PPE and safe operating practices [3].

\section{H. S. Tilney}

henry.tilney@nhs.net

1 Department of Colorectal Surgery, Frimley Park Hospital, Portsmouth Road, Surrey, Camberley GU16 7UJ, UK

2 Department of Surgery, The Royal Marsden Hospital, London, UK

3 Inspiral Health, St Luke's House, London, UK
The restructuring of surgical pathways as part of the response to COVID has, in our experience as well as commentary from this journal [4], led to further expansion of units dedicated to clean, elective surgery. The time that staff have been able to dedicate to these patients has rationalised their care and led to improved short-term outcomes including a reduced length of stay and high patient satisfaction [5]. A pause to elective treatment was also introduced during the initial surge in COVID cases at Frimley Park Hospital. Prioritisation of cases was then performed according to clinical factors (including obstructive symptoms) and adverse radiological features (such as evidence of extra-mural vascular invasion). Doing so allowed the debate over minimally invasive colorectal surgery in the context of COVID to mature, with relaxation of the concerns regarding minimally invasive surgery. In view of this, with appropriate precautions, laparoscopic surgery has remained our default position for colonic resection, and robotics the default for rectal cancer surgery. Our indications for the use of defunctioning stomas have not changed, and in no cases has our operative strategy changed to perform a non-restorative procedure due to the perceived increased risks in the COVID era. This is in contrast to the experience of some authors internationally [1] and in spite of initial UK guidance cautioning against both minimally invasive surgery and restorative surgery [2].

In summary, we believe that the use of minimally invasive surgery, in a 'clean' unit capable of flexing to the needs of the hospital in response to COVID surges offers the best paradigm for the surgical management of colorectal cancer, and other urgent colorectal conditions, in the current pandemic.

\section{Compliance with ethical standards}

Conflict of interest The authors declare that they have no conflict of interest.

Ethical approval Nil relevant as no patient level data presented.

Informed consent For this type of study formal consent is not required. 


\section{References}

1. Yao H, Pang K, Xiao G et al (2020) What should surgeons do in face of the COVID-19 pandemic? A Beijing experience. Dis Colon Rectum. https://doi.org/10.1097/DCR.000000000000172 6 (published online ahead of print, 13 May 2020)

2. Updated intercollegiate general surgery guidance on COVID-19. https://www.acpgbi.org.uk/content/uploads/2020/03/UpdatedIntercollegiate-General-Surgery-Guidance-on-COVID-19-final -with-logos13.pdf. Accessed 2 July 2020

3. Soliman $\mathrm{M}$ (2020) Controversies in $\mathrm{CO}_{2}$ insufflation and COVID19. Tech Coloproctol 24:667-670. https://doi.org/10.1007/s1015 1-020-02237-2

4. Gallo G, La Torre M, Pietroletti R et al (2020) Italian society of colorectal surgery recommendations for good clinical practice in colorectal surgery during the novel coronavirus pandemic. Tech Coloproctol 24:501-505. https://doi.org/10.1007/s10151-02002209-6

5. Huddy JR, Freeman Z, Crockett M et al (2020) Establishing a "cold" elective unit for robotic colorectal and urological cancer surgery and regional vascular surgery following the initial COVID-19 surge. Br J Surg. https://doi.org/10.1002/bjs.11922

Publisher's Note Springer Nature remains neutral with regard to jurisdictional claims in published maps and institutional affiliations. 